\title{
Variable Weight Code Division Multiple Access System for Monitoring Vibration of Unequally Distributed Points
}

\author{
Saleh Seyedzadeh, Member, IEEE, Ivan Glesk, Senior Member, IEEE, Farzad Pour Rahimian*, and \\ Wing C. Kwong**, Senior Member, IEEE \\ Faculty of Engineering, University of Strathclyde, Glasgow G1 1XW, UK. \\ ${ }^{*}$ Faculty of Engineering \& Environment, Northumbria University, Newcastle, UK. \\ ** Department of Engineering, Hofstra University, Hempstead, USA.
}

\begin{abstract}
The ever-growing demand for more accurate structural health monitoring of large scale facilities such as modern high-speed railways and bridges have resulted in the development of optical sensor networks (OSN), which help eliminate the disadvantages of conventional electric sensors, the most significant of which are sensitivity to electromagnetic interferences and larger sizes. The existing fibre optic infrastructures that are implanted mainly for communication purposes are not widely used by OSNs, due to the lack of appropriate multiplexing techniques. This study proposes an optical code division multiple access (OCDMA) system for support vibration sensing of unequally distributed points. The proposed system takes the advantages of spectral amplitude encoding (SAC) technique in providing differentiated services in physical layer by varying code weights. Simulation results monitoring three vibration sensor nodes with different distances are presented in the paper. The simulation and mathematical analysis indicate the suitability and simple implementation of the proposed system for supporting vibration sensing with high accuracy.
\end{abstract}

Keywords: Structural Health Monitoring, Vibration Sensing, Optical Sensing, Optical Code Division Multiple Access, Unequally Distributed Sensor Nodes

\section{INTRODUCTION}

The advantages of optical sensors such as small size of optical sensors, accuracy and immunity to electromagnetic noise have made a better solution in structural health monitoring (SHM) [1]. Lately, the use of optical multiplexing techniques has improved the efficiency of optical sensing for distributed sensor network in terms of cost and system complexity [2]. Fibre optic sensing has been implemented as intrinsic in which the fibre itself is used as the sensing element or extrinsic as a medium to communicate the sensed signal. The use of optical sensors in construction industry for monitoring gas leakage, temperature, strain, reinforced concrete beam and building cladding systems [3].

Vibration is essential for the health monitoring on civil structures to evaluate the structural condition and to identify the internal damages at an early stage [2]. The use of traditional electric sensors in measuring vibration high temperatures and sensors are noticeably exposed to electromagnetic interference; hence, they can be utilised for single-point simplex measuring. To deal with this issue, different optical multiplexing techniques have been proposed for optical vibration sensing of distributed points. These include time division multiplexing, wavelength division multiplexing, frequency modulated carrier wave, and optical code division multiple access [3, 4].

Due to the problems with the mentioned techniques such as cost, low SNR and scanning time and need for synchronisation, Optical Code Division Multiple Access (OCDMA) sensor network using synchronous and asynchronous techniques was proposed [5]. The asynchronous OCDMA technique namely spectral amplitude coding (SAC) has satisfied the requirement among other OCDMAs due to better multiple access interference (MAI) cancellation and simplicity of implementation [6]. Majority of researches have focused on equally distributed sensor points where the received optical power (ROP) for each sensor is supposed to be similar. However; in SHM most structures are distributed with different distances, and it is not possible to setup the network based in a place having equal distance with all sensing point; hence; a further power loss will be imposed for ROP of signals related to farther points. It should be noted that the use of optical amplifiers increases the noise as well as the cost. Recently, variable-weight SAC (VW-SAC) system have been proposed to support differentiated quality of service (QoS) in optical communication networks [7, 8]. In VW-SAC higher weight codes give better signal quality due to more wavelengths being assigned to a particular user or service. Different code families [7, 8, 9, 10] and detection techniques [11] have been proposed for VW-SAC system and successful experimental proof for optical communication has been reported [12]. 
In this paper, a VW-SAC system is proposed for quasi-distributed vibration sensing to maintain the deliverable signals for all nods in the network. The proposed system eliminates the demand for in-line amplification and thereby eases the implementation, expansion and maintenance of the network. In this system, the higher weights are allocated for the nodes having longer distance from the system base.

\section{VW-SAC CODE AND ARCHITECTURE}

In OCDMA systems, all users have asynchronous access to the medium, which is achieved by assigning each particular user with a specific code signature. This is achieved by assigning each particular user with a specific code signature, which distinguishes one user from the others. At the receiver side, each corresponding decoder can recover the data sequence and signal from other users is enumerated as multiple access interference (MAI). Among several VW-code families, we used VW Khazani-Syed (VW-KS) code which was developed based on the single weight KS code [7]. KS code is based on matrix construction, in which two sub-codes $A=[110]$ and $B=[011]$ are used to construct the basic matrix. The structure of this code is in such a way that cross-correlation (the number of overlapping chip(s) between two different users' codes), $R$, between each pair of different users' codes is zero or one which results in reduction of MAI effect. The VW-KS code construction consists of a combination of two algorithms, which are fixed mapping technique and dynamic weight assignment. The first technique is a simple mapping of codes with different weights that had been initially constructed using single weight KS technique. The second technique, dynamic weight assignment uses the value of [121] combination to arrange sub-codes A and B, accordingly. The main aim of developing dynamic weight assignment was to support few number of differentiated services without wasting extra bandwidth.

We assume that sensing points are distributed in different zones, in which the nodes inside same zone have almost the same distance from base control unit and different zones are located unequally from the base control unit. Figure 1 shows three zones with different sensing nodes and the base control unit. The main idea of this work is to assign a specific code weight for each zone where higher weights are allocated for zones with farther distance from base. In this case, nodes are categorised based on their distances into zones, which is equivalent with services in communication systems.

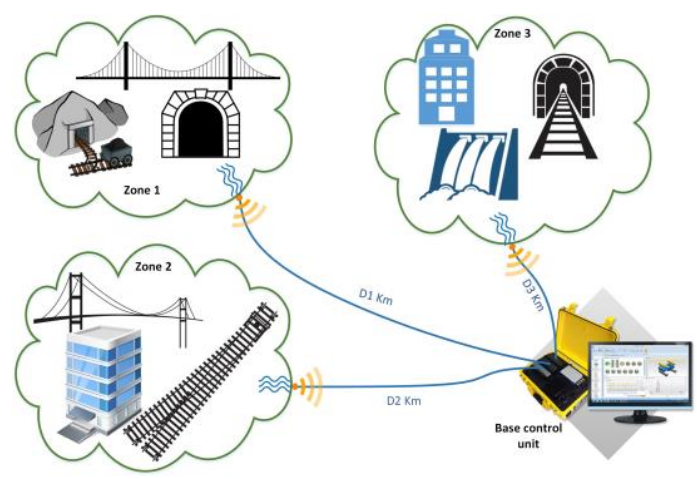

Figure 1. Configuration of distributed optical sensors as zones.

Nine code-words are generated using VW-KS code with chip spacing of $0.4 \mathrm{~nm}$ and code length of 30 . The range of optical frequencies varies from 192.2 to $193.65 \mathrm{THz}$, as shown in Figure 2. Two points in the nearest zone with the allocated weight of 2 , three points in zone 2 with the weight of 4 , and four-point at the farthest zone with weight 6 are considered.

\begin{tabular}{|c|c|c|c|c|c|c|c|c|c|c|c|c|c|c|c|c|c|c|c|c|c|c|c|c|c|c|c|c|c|c|c|}
\hline \multirow[b]{2}{*}{$\begin{array}{l}\# \\
\text { \#̈ } \\
\dot{\mathscr{S}}\end{array}$} & \multirow[b]{2}{*}{ 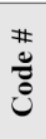 } & \multicolumn{18}{|c|}{$W=6$} & \multicolumn{9}{|c|}{$W=4$} & \multicolumn{3}{|c|}{$\mathbf{W}=\mathbf{2}$} \\
\hline & & $\begin{array}{l}\stackrel{N}{ } \\
\stackrel{2}{\leftarrow}\end{array}$ & $\begin{array}{l}\stackrel{\text { N }}{\mathrm{N}} \\
\text { Oె }\end{array}$ & 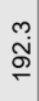 & $\begin{array}{l}\text { ભ } \\
\text { నू } \\
\stackrel{2}{-}\end{array}$ & $\stackrel{+}{\stackrel{+}{\sigma}}$ & $\begin{array}{l}\stackrel{2}{+} \\
\stackrel{2}{\sim} \\
\stackrel{2}{\leftarrow}\end{array}$ & $\begin{array}{l}\stackrel{n}{N} \\
\stackrel{2}{\sigma}\end{array}$ & 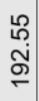 & $\begin{array}{l}\stackrel{0}{ } \\
\text { } \\
\stackrel{2}{-}\end{array}$ & 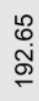 & ָָ & $\begin{array}{l}\stackrel{\circ}{\sim} \\
\stackrel{2}{\sim} \\
\stackrel{2}{-}\end{array}$ & $\begin{array}{l}\infty \\
\text { ก̃ } \\
\stackrel{2}{-}\end{array}$ & $\begin{array}{l}\stackrel{2}{\infty} \\
\text { ஸ் } \\
\stackrel{\sigma}{\leftarrow}\end{array}$ & $\begin{array}{l}\text { ๙ેं } \\
\stackrel{\sigma}{\sigma}\end{array}$ & 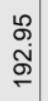 & $\stackrel{\mathscr{\Omega}}{-}$ & 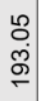 & $\stackrel{\check{g}}{\sigma}$ & $\begin{array}{l}\stackrel{\omega}{.} \\
\stackrel{3}{\circ}\end{array}$ & $\begin{array}{l}\stackrel{N}{~} \\
\stackrel{\text { Oे }}{\sigma}\end{array}$ & $\begin{array}{l}\stackrel{\text { N }}{\text { ஜ் }} \\
\stackrel{\sigma}{\leftarrow}\end{array}$ & 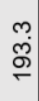 & 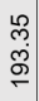 & 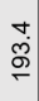 & $\begin{array}{l}\stackrel{\circ}{\sim} \\
\stackrel{\sigma}{-}\end{array}$ & 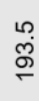 & $\begin{array}{l}\text { مீ } \\
\text { ஜூ }\end{array}$ & 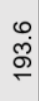 & $\begin{array}{l}\text { மீ } \\
\text { ஜு }\end{array}$ \\
\hline \multirow{3}{*}{1} & $\mathrm{C}_{1}$ & 1 & 1 & 0 & 1 & 1 & 0 & 1 & 1 & 0 & 0 & 0 & 0 & 0 & 0 & 0 & 0 & 0 & 0 & 0 & 0 & 0 & 0 & 0 & 0 & 0 & 0 & 0 & 0 & 0 & 0 \\
\hline & $\mathrm{C}_{2}$ & 0 & 1 & 1 & 0 & 0 & 0 & 0 & 0 & 0 & 1 & 1 & 0 & 1 & 1 & 0 & 0 & 0 & 0 & 0 & 0 & 0 & 0 & 0 & 0 & 0 & 0 & 0 & 0 & 0 & 0 \\
\hline & $\mathrm{C}_{3}$ & 0 & 0 & 0 & 0 & 1 & 1 & 0 & 0 & 0 & 0 & 1 & 1 & 0 & 0 & 0 & 1 & 1 & 0 & 0 & 0 & 0 & 0 & 0 & 0 & 0 & 0 & 0 & 0 & 0 & 0 \\
\hline \multirow{3}{*}{2} & $\mathrm{C}_{5}$ & 0 & 0 & 0 & 0 & 0 & 0 & 0 & 0 & 0 & 0 & 0 & 0 & 0 & 0 & 0 & 0 & 0 & 0 & 1 & 1 & 0 & 1 & 1 & 0 & 0 & 0 & 0 & 0 & 0 & 0 \\
\hline & $\mathrm{C}_{6}$ & 0 & 0 & 0 & 0 & 0 & 0 & 0 & 0 & 0 & 0 & 0 & 0 & 0 & 0 & 0 & 0 & 0 & 0 & 0 & 1 & 1 & 0 & 0 & 0 & 1 & 1 & 0 & 0 & 0 & 0 \\
\hline & $\mathrm{C}_{7}$ & 0 & 0 & 0 & 0 & 0 & 0 & 0 & 0 & 0 & 0 & 0 & 0 & 0 & 0 & 0 & 0 & 0 & 0 & 0 & 0 & 0 & 0 & 1 & 1 & 0 & 1 & 1 & 0 & 0 & 0 \\
\hline \multirow{2}{*}{3} & $\mathrm{C}_{8}$ & 0 & 0 & 0 & 0 & 0 & 0 & 0 & 0 & 0 & 0 & 0 & 0 & 0 & 0 & 0 & 0 & 0 & 0 & 0 & 0 & 0 & 0 & 0 & 0 & 0 & 0 & 0 & 1 & 1 & 0 \\
\hline & $\mathrm{C}_{9}$ & 0 & 0 & 0 & 0 & 0 & 0 & 0 & 0 & 0 & 0 & 0 & 0 & 0 & 0 & 0 & 0 & 0 & 0 & 0 & 0 & 0 & 0 & 0 & 0 & 0 & 0 & 0 & 0 & 1 & 1 \\
\hline
\end{tabular}

Figure 2. Code construction for vibration system and the frequencies for each chip in OCDMA system. 


\section{SYSTEM DESCRIPTION}

Figure 3 shows the (proof-of-concept) architecture of a VW-SAC OCDMA system designed for three zones. Amplified spontaneous emission broad-band source is used as the optical source. The spectrum of BBS is sent through an optical circulator and a $1 \times 3$ optical coupler. Each port of coupler is then connected to sensor point using single mode fibre (SMF) with different lengths of 8,12 and $15 \mathrm{~km}$. The encoder of VW-SAC comprises of a collimator, vibration box and a series of FBGs. Due to the property of VW-KS code in being double weighted the two wavelengths can be reflected using one FBG with bandwidth twice the chip spacing. The generated vibration cause a modulation that can be detected by decoders. In our simulation set-up, we used Mach-Zehender modulator to mimic the behaviour of collimator in sensing vibration. Three different (radio) frequencies of 70, 140, and 210 $\mathrm{MHz}$ are used to represent low, medium and high vibration, respectively.

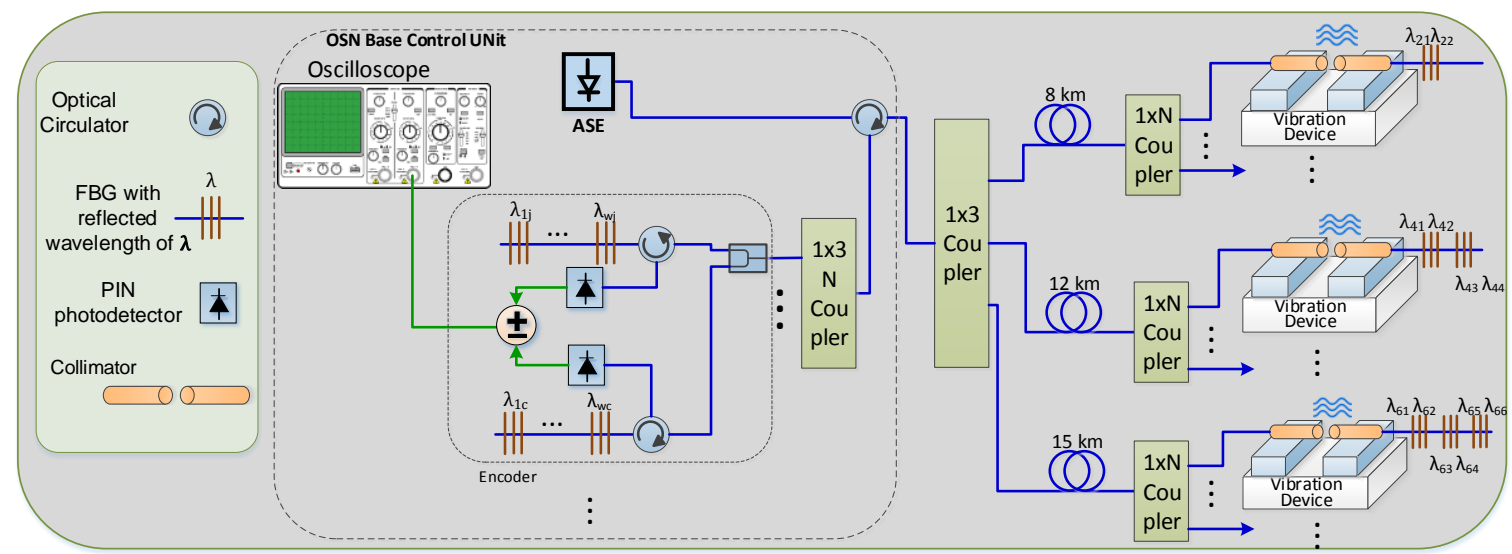

Figure 3. VW-SAC OCDMA system structure of fibre vibration sensor for monitoring of three zones.

The modulated signals are then travelled back to the coupler, combined and then guided to the detection section by circulator. Here, the combined signals are split among decoders using a coupler. FBG are also used to filter the desire wavelengths. This study used conventional complementary subtraction detection [4, 13]. It uses balanced detection to eliminate the effect of MAI. The upper decoder has the same structure as the encoder at the transmitter side, while the lower decoder is the complement of the upper decoder. In order to prevent power reduction of various chips, the FBGs with bandwidth of $0.4 \mathrm{~nm}$ arranged in opposite order of the encoder. This technique also eliminates the delay imposed by FBGs at the decoder. For simplification purpose, only one sensing point in each zone and one decoder is shown in the figure. The decoded optical signals are finally converted into electrical domain by means of PIN photodectectors and sent to three channel oscilloscope for be translated using fast Fourier transform.

\section{SIMULATION RESULTS}

Three different vibration scenarios is considered to demonstrate the performance of system and the output of sensing point with lowest SNR in each zone is depicted. These nodes namely N1, N2, and N3 are located 8, 12, and $15 \mathrm{~km}$ far from the base control unit, respectively. Figures 4(a) to 4(c) illustrate the power in radio frequency (RF) domain of three nodes for different scenarios. As it can be observed low, medium and high vibration signal are constantly retrieved at frequencies of $68.4,141.2$, and $209.9 \mathrm{MHz}$ at the encoder. Moreover, the signals of all nodes in different configurations are revived at almost the same peak power. The configuration of utilised weights are based on the selected distances and different combination can be applied for desired results.

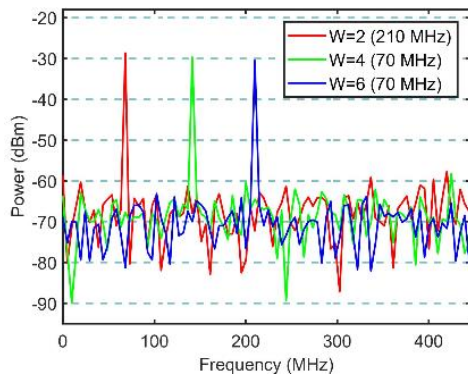

(a)

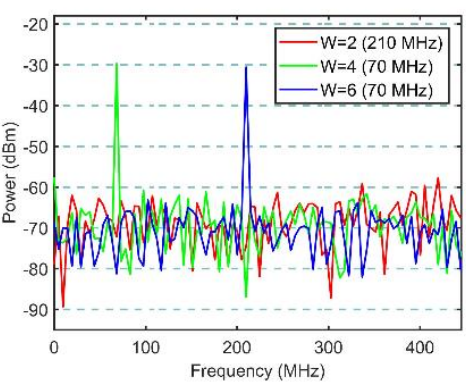

(b)

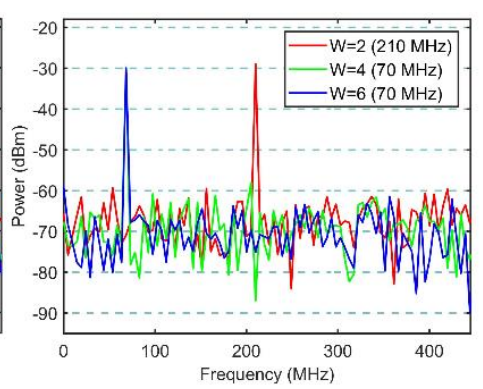

(c)

Figure 4. The RF signal power received for three nodes in each zones. 
Finally, the fibre length for all nodes is set to $20 \mathrm{~km}$. Figure 5 depicts the signal power for different frequencies. The signal for three nodes are received at different levels, where it was impossible to recognise vibrations happening in node N1. This is due to the fact that the received optical power of that nodes are lower than others.

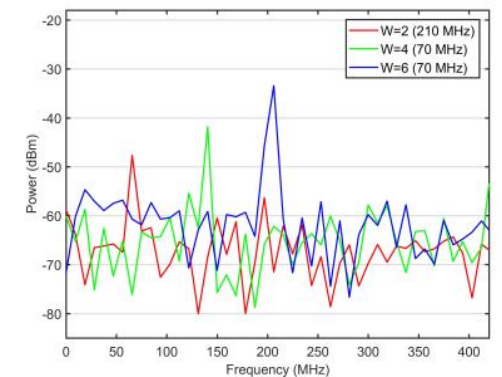

Figure 5. The RF signal power received for distance of $20 \mathrm{~km}$ for all nodes.

\section{CONCLUSION}

An optical multiplexing system for monitoring vibration in unequally distributed nodes was proposed based on VW-SAC OCDMA system. The proposed system with three nodes with different distances from control unit was investigated. The results indicated that the applied vibration frequencies was explicitly obtained for all weights in various scenarios. The performance of system may be further improved using more flexible OCDMA code families.

\section{ACKNOWLEDGMENT}

This project has received funding from the European Union's Horizon 2020 research and innovation program under the Marie Sklodowska-Curie grant agreement No 734331. It is also supported in part by the Faulty Development and Research Grants of Hofstra University.

\section{REFERENCES}

[1] M. Majumder et al.: Fibre Bragg gratings in structural health monitoring-Present status and applications, sep 2008.

[2] H. N. Li et al.: Recent applications of fiber optic sensors to health monitoring in civil engineering, Engineering Structures, vol. 26, pp. 1647-1657, sep 2004.

[3] E. Udd and W. B. Spillman Jr: Fiber optic sensors: an introduction for engineers and scientists. 2011.

[4] W.C. Kwong and G.-C. Yang: Optical Coding Theory with Prime, New York: CRC Press, 2013.

[5] J. Ko, et al:: "Fiber Bragg grating sensor network based on code division multiple access using a reflective semiconductor optical amplifier," Microwave and Optical Technology Letters, vol. 52, pp. 378-381, 2010.

[6] A. Noura et al:: Simultaneous vibration and humidity measurement using a hybrid WDM/OCDMA sensor network, in 4th International Conference on Photonics, ICP 2013 - Conference Proceeding, pp. 163-165, IEEE, 2013.

[7] S. B. A. Anas, et al.: Variable weight Khazani-Syed code using hybrid fixed-dynamic technique for optical code division multiple access system, Optical Engineering, vol. 55, p. 106101, 2016.

[8] S. Seyedzadeh et al.: Variable weight spectral amplitude coding for multiservice OCDMA networks, Optical Fiber Technology, vol. 37, pp. 53-60, 2017.

[9] W. Kwong and G.-C. Yang: Multiple-Length Multiple-Wavelength Optical Orthogonal Codes for Optical CDMA Systems Supporting Multirate Multimedia Services, IEEE Journal on Selected Areas in Communications, vol. 22, pp. 1640-1647, 2004.

[10] W. Liang et al: A new family of 2D variable-weight optical orthogonal codes for OCDMA systems supporting multiple QoS and analysis of its performance, Photonic Network Communications, vol. 16, pp. 53-60, 2008.

[11] S. Seyedzadeh et al.: Variable-weight optical code division multiple access system using different detection schemes," Journal of Telecommunications and Information Technology, vol. 2016, no. 3, 2016.

[12] S. Seyedzadeh et al:: Experimental demonstration of variable weight SAC-OCDMA system for QoS differentiation, Optical Fiber Technology, vol. 20, pp. 495-500, 2014.

[13] S. Seyedzadeh et al.: Weight optimization of variable weight OCDMA for triple-play services," in 4th International Conference on Photonics, ICP 2013 - Conference Proceeding, pp. 99-101, IEEE, 2013. 